\title{
Accelerated Monte Carlo system reliability analysis through machine-learning-based surrogate models of network connectivity
}

\author{
R. E. Stern ${ }^{\mathrm{a}}$, J. Song ${ }^{\mathrm{b}, *}$, D. B. Work ${ }^{\mathrm{a}}$ \\ ${ }^{a}$ University of Illinois at Urbana-Champaign \\ ${ }^{b}$ Seoul National University
}

\begin{abstract}
The two-terminal reliability problem in system reliability analysis is known to be computationally intractable for large infrastructure graphs. Monte Carlo techniques can estimate the probability of a disconnection between two points in a network by selecting a representative sample of network component failure realizations and determining the sourceterminal connectivity of each realization. To reduce the runtime required for the Monte Carlo approximation, this article proposes an approximate framework in which the connectivity check of each sample is estimated using a machinelearning-based classifier. The framework is implemented using both a support vector machine (SVM) and a logistic regression based surrogate model. Numerical experiments are performed on the California gas distribution network using the epicenter and magnitude of the 1989 Loma Prieta earthquake as well as randomly-generated earthquakes. It is shown that the SVM and logistic regression surrogate models are able to predict network connectivity with accuracies of $99 \%$ for both methods, and are 1-2 orders of magnitude faster than using a Monte Carlo method with an exact connectivity check.
\end{abstract}

\section{Introduction}

In the aftermath of a natural disaster, a prompt response is critical to minimize economic damage and the loss of life $[1,2]$. In order to improve post-disaster response times to hazards that impact lifeline networks such as gas, water, and electricity distribution systems, efficient methods are needed to quickly and accurately estimate the system-level network failure probability under a given set of individual component failure probabilities before crews have time to inspect the extent of the damage. This will enable first responders to make strategic and immediate risk-informed decisions about relief efforts before there is time to assess damage to the infrastructure based on comprehensive and visual inspections.

System reliability analysis (SRA) of infrastructure networks encompasses a number of methods to determine the probability that a network will be able to complete its designed function after a disaster event. The methods are used to analyze the resilience of the network with respect to failure under a catastrophic event $[3,4,5,6,7]$.

To apply an SRA method, the infrastructure network is first modeled as a graph $\mathcal{G}=(\mathcal{V}, \mathcal{E})$ where $\mathcal{V}$ is the vertex (node) set and $\mathcal{E} \subseteq\{(i, j): i, j \in \mathcal{V}\}$ is the set of edges with cardinality $n=|\mathcal{E}|$. This network is a stochastic network where the $i^{t h}$ edge fails randomly with probability $p_{f, i}$.

Given a network $\mathcal{G}$ and a vector of the correlated edge failure probabilities $p_{f}=\left[p_{f, 1}, \cdots, p_{f, n}\right]^{T}$, the two-terminal

\footnotetext{
* Corresponding author
}

Email address: junhosong@snu.ac.kr (J. Song) reliability problem [8] is to find the probability that a connection (i.e., a path) remains between a source node $s \in \mathcal{V}$ and a terminal node $t \in \mathcal{V}$. This problem has been studied extensively for infrastructure, communications, and wireless networks $[9,10,11,12,13,14,15]$.

For some special cases of graphs, the two-terminal reliability problem can be efficiently solved. For graphs consisting of series and parallel subgraphs, as well as graphs with a bounded path width (a measure of the decomposition of a graph), decomposition algorithms exist to solve reliability problems in linear time $[16,17]$. However, in its most general form, the two-terminal reliability problem is $\sharp \mathcal{P}$-complete $[18,19]$, which is a complexity class of problems that involve counting the number of solutions to a problem in $\mathcal{N} \mathcal{P}$. No exact polynomial-time algorithms are known to solve problems in $\sharp \mathcal{P}$-complete, which motivates the use of approximate or heuristic approaches.

One popular approximation approach is to use Monte Carlo simulation (MCS) to approximate the probability of disconnection. In this approach, a damaged network realization is simulated by removing edges in the network according to their failure probability. The connectivity of $s$ and $t$ are checked on each network realization, and the probability of disconnection of $s$ and $t$ is estimated as the fraction of disconnected samples compared to the total sample size. A bound on the number of samples required to achieve a converged estimate of the failure probability within a given error bound is provided by Karger [20].

To evaluate the connectivity of each realization, several exact methods exist $[21,22,23,24,25]$. However, these 
methods can be time-consuming for large infrastructure networks.

In order to reduce the computation time associated with each connectivity check, this article proposes the use of a constant-time surrogate model for network connectivity to approximate whether $s$ and $t$ are connected in each network realization of the MCS. Specifically, we address the question of how to construct a machine-learning-based surrogate model for network connectivity.

We develop an approach to estimate whether a source and terminal node in the network are connected through a supervised machine-learning-based classifier using support vector machines (SVM) with a non-linear (Gaussian) kernel. SVM is a popular machine learning classifier that has been applied in previous work as a surrogate model for the component limit-sate function in MCS-based SRA $[26,27]$. However, to the best of our knowledge, this is the first time SVM is being used to estimate the probability of network failure. We also develop a surrogate model for network connectivity using the popular logistic regression classifier, and compare the results. Logistic regression uses a linear kernel, and is included for comparison and to better understand the limitations of such a method as a surrogate model. In an earlier version of this work [28], the machine-learning algorithm AdaBoost [29] is used as a surrogate model for network connectivity. However, AdaBoost was found to preform poorly on data sets with a class imbalance (e.g., predominantly realizations corresponding to network survival), while logistic regression and kernel SVM offer improved performance.

The estimated network reliability depends heavily on statistical dependencies of component failures [30]. In this article, we develop a surrogate model that performs well even with correlated edge failures.

The primary contribution of this article is to accelerate MCS for estimating the network failure probability by developing a machine-learning-based surrogate model for node connectivity. This surrogate model is learned from examples of network failure, and is not learned using specific component failure probabilities. This is intended to make the the model applicable to a broad range of hazard scenarios.

This article demonstrates that machine-learning-based classifiers such as logistic regression and non-linear SVM can learn network structure. They can be used to determine source-terminal connectivity based on individual edge failures, which can also be integrated into the traditional MCS framework for SRA.

The remainder of this article is outlined as follows. The Monte Carlo framework as well as surrogate models for network connectivity are described in Section 2. Numerical examples with correlated, non-uniform edge failures are considered in Section 3. Finally, we conclude that surrogate modeling via machine learning is a promising alternative to current SRA techniques and propose further work in Section 4.

\section{Approximate System Reliability Analysis}

\subsection{Monte Carlo framework}

To conduct MCS for network reliability we first define the network state. Given an infrastructure graph $\mathcal{G}$, the network state following an event $E$ is an $n$-dimensional binary random variable $X=\left[X_{1}, \cdots, X_{n}\right]^{T}$, with elements $X_{i}$ defined as

$$
X_{i}= \begin{cases}0 & \text { with probability } 1-p_{f, i} \\ 1 & \text { with probability } p_{f, i}\end{cases}
$$

where individual component failure probabilities, $p_{f, i}$ may be correlated.

Individual network realizations $x \in\{0,1\}^{n}$ can be drawn from this marginal distribution where each element $x_{i}$ encodes the state of the corresponding edge as

$$
x_{i}= \begin{cases}0 & \text { if edge } i \text { is intact } \\ 1 & \text { if edge } i \text { has failed }\end{cases}
$$

Suppose $K$ samples are drawn from the distribution (1) using any desired sampling method, the $k^{t h}$ network state is denoted $x(k)$.

The network connectivity status $Y_{s, t}$ is a scalar binary random variable defined with respect to source node $s$ and terminal node $t$ as follows. $Y_{s, t}=0$ with probability equal to the probability at least one path exists between $s$ and $t$, and $Y_{s, t}=1$ otherwise. Individual samples $y_{s, t}(k)$ of $Y_{s, t}$ can be computed from samples $x(k)$ of $X$ as:

$$
y_{s, t}(k)= \begin{cases}0 & \text { if } s \text { and } t \text { are connected in } x(k) \\ 1 & \text { otherwise. }\end{cases}
$$

For each sampled network realization $x$ the source-terminal connectivity $y_{s, t}$ of the realization can be directly computed since the component states are deterministic in the given sample.

The quantity of interest in SRA is the probability that nodes $s$ and $t$ are disconnected following an event $E$, denoted $P\left(Y_{s, t}=1 \mid E\right)$. This notation is simplified by implicitly conditioning on hazard scenario $E$ as $P\left(Y_{s, t}=1\right)$. We use MCS with $K$ samples to estimate the probability of network failure as:

$$
P\left(Y_{s, t}=1\right) \approx \hat{P}\left(Y_{s, t}=1\right)=\frac{1}{K} \sum_{k=1}^{K} y_{s, t}(k),
$$

where $\hat{P}\left(Y_{s, t}=1\right)$ denotes the MCS estimate.

Karp, et al. [31] proved that computing

$$
K=\mathcal{O}\left(\frac{\log n}{\varepsilon^{2} \cdot P\left(Y_{s, t}=1\right)}\right)
$$

realizations of the MCS provides an estimate for the network failure probability $P\left(Y_{s, t}=1\right)$ accurate to within $\varepsilon$ with high probability, where $\varepsilon$ is the desired maximum 


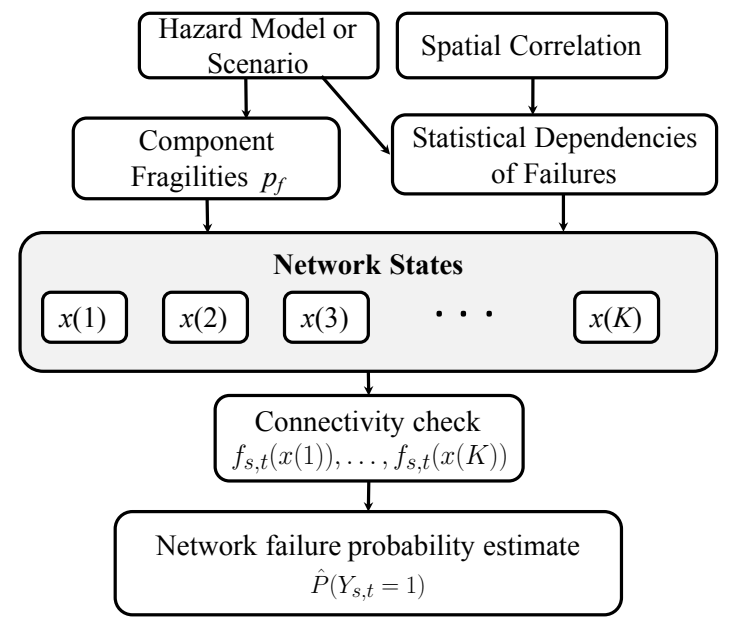

Figure 1: General MCS framework for computing the probability of network failure.

error in estimation. As it is defined, this requires prior knowledge on the network failure probability. However, this provides valuable insight into the relationship between the rarity of network failure and the number of MCS realizations required to obtain a reliable estimate on the network failure probability. An alternative stopping criterion is to use the coefficient of variation (COV) for MCS, which is defined in terms of the estimated network failure probability.

The general framework for the MCS method of estimating the network failure probability is outlined in Figure 1. Here a hazard model and the spatial correlation of component failures are used to simulate a disaster scenario and compute the failure probability vector $p_{f}$. These edge failure probabilities are used to generate a set of network realizations $\{x(1), \ldots, x(K)\}$. For each realization $x(k)$, a connectivity check is conducted using a connectivity function

$$
f_{s, t}(\cdot)= \begin{cases}0 & \text { if } s \text { and } t \text { are connected } \\ 1 & \text { if a network failure has occurred, }\end{cases}
$$

and the connectivity results are aggregated to compute the overall network failure probability estimate, $\hat{P}\left(Y_{s, t}=1\right)$ according to (4).

Finally, the COV of the network failure probability estimate $\delta$ is computed by:

$$
\delta=\frac{s_{K}}{\sqrt{K} \cdot \hat{P}\left(Y_{s, t}=1\right)},
$$

where

$$
s_{K}=\sqrt{\frac{1}{K-1} \sum_{k=1}^{K}\left(f_{s, t}(x(k))-\hat{P}\left(Y_{s, t}=1\right)\right)^{2}}
$$

is the sample standard deviation of network failure prob- ability estimates, $K$ is the total number of MCS, and $\hat{P}\left(Y_{s, t}=1\right)$ is the network failure probability estimate.

\subsection{Exact source-terminal connectivity}

To estimate the network failure probability via MCS, the source-terminal connectivity of each network realization must be determined. A popular and efficient algorithm to check the connectivity of $s$ and $t$ is depth first search (DFS) [32, 25, 33].

Depth first search is a recursive algorithm that begins at a source node and explores along a path by following an edge to a neighboring node until there are no more nodes to be visited along that path. When this occurs, the algorithm backtracks to the last node with at least one unvisited neighboring node, and creates a new path in the direction of the unvisited neighbor. The new path continues until no new unvisited nodes are found, at which point the backtracking process is repeated. This process is continued until all possible nodes have been reached. If the terminal node has not been reached after the algorithm terminates, the source and terminal nodes are found to be disconnected.

The runtime complexity of DFS is $\mathcal{O}(|\mathcal{E}|+|\mathcal{V}|)[22]$. This is significantly faster than shortest path algorithms such as Dijkstra's algorithm [23], which requires $\mathcal{O}\left(|\mathcal{V}|^{2}\right)$ to compute the shortest path to check connectivity between any two nodes. This makes DFS a natural choice for quickly checking connectivity between two nodes. However, DFS is still a linear-time algorithm, and thus does not scale as well as a constant-time algorithm enabled by the use of a surrogate model.

\subsection{Surrogate models for vertex connectivity}

To quickly estimate the probability of network failure, a constant-time connectivity check is preferable to one that operates in linear time. For large networks we introduce the idea of constructing a surrogate model for network connectivity by predicting network behavior with a machinelearning-based classifier. This allows for quickly determining whether two nodes are connected, but introduces some error due to the imprecise nature of surrogate models.

The resulting framework for SRA using a machinelearning-based surrogate model for checking connectivity is shown in Figure 2, and consists of two phases. In the first phase, a surrogate model is trained off-line by generating training data of example network failures. The training data consists of network realizations and corresponding true class labels, and is used to learn a surrogate model $\vec{f}(\cdot)$ for $(6)$. The MCS SRA step is executed after the model has been trained. The procedure for estimating the network failure probability using the surrogate model is the same as in Figure 1 with the exception that instead of exactly computing connectivity, an approximate check is made using the learned surrogate model $\widetilde{f}(\cdot)$. A potential drawback is that if biased training data set is selected, it 


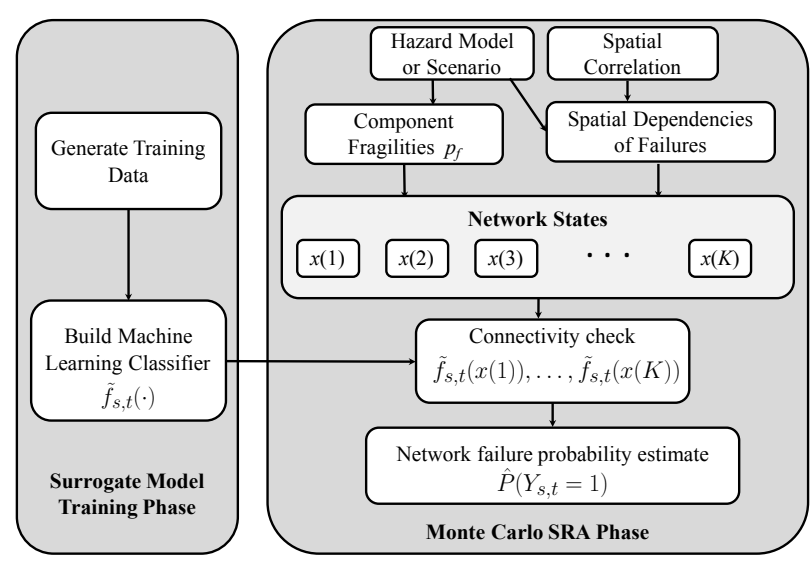

Figure 2: Framework for approximate SRA using MCS with surrogate model.

is possible for the surrogate model to exhibit a bias in prediction. Next, we introduce two machine learning methods to construct a network connectivity surrogate model.

\subsection{Classification with kernel support vector machines}

Let $\mathcal{X}=\{x(1), \cdots, x(p)\}$ denote a feature set of $p$ state realizations, with corresponding source terminal connectivity given in the label set $\mathcal{Y}=\{y(1), \cdots, y(p)\}$. In supervised machine learning classification problems, a training dataset $\mathcal{D}_{\mathrm{tr}}=\{\mathcal{X}, \mathcal{Y}\}$ is given to the algorithm, such that the relationship between the features and the class labels (e.g., connected or disconnected) can be learned. One method to learn the relationship is through the construction of a support vector machine [34].

To apply the SVM technique, a slight relabeling of the connectivity class labels from $\{0,1\}$ to $\{-1,1\}$ is required. On the relabeled data, the linear support vector classifier is of the form:

$$
\widetilde{f}(x)=\operatorname{sign}\left(\sum_{k=1}^{p} \alpha_{k} y(k) x(k)^{T} x+b\right)
$$

where $\alpha_{k}$ are the parameters to be learned, and it returns the SVM class labels of $\{-1,1\}$. A minor relabeling of the outcomes (e.g., $-1 \rightarrow 0$ ) yields a predictor for the network connectivity state in the original class labels. Note that this SVM classifier is constructed with a binary feature space instead of the more common continuous feature space. For more details on SVM with binary features we refer the reader to [35]. When the classes are linearly separable in the $n$-dimensional space of the feature vector, the learned classifier can achieve exact classification on the training data, otherwise a measure of the misclassification error is minimized. When the training data is not linearly separable, an alternative strategy is to map the training data into a much higher dimensional feature vector denoted by $\Phi(x)$, and look for a classifier in the higher dimensional space. The new classifier becomes

$$
\widetilde{f}(x)=\operatorname{sign}\left(\sum_{k=1}^{p} \alpha_{k} y(k) \Phi(x(k))^{T} \Phi(x)+b\right) .
$$

Interestingly, it is not necessary to explicitly define the mapping to the high dimensional space, since only the inner product $\Phi^{T} \Phi$ is needed in the classifier. The inner product can instead be defined through a kernel function $K(x(k), x(j))=\Phi(x(k))^{T} \Phi(x(j))$. The use of the kernel function directly in (9) is known as the kernel trick [36] in machine learning. In the present work, we adopt an SVM Gaussian kernel of the form:

$$
K(x(k), x(j))=\exp \left(-\frac{1}{2 \sigma}\|x(k)-x(j)\|_{2}^{2}\right),
$$

where $\sigma$ is a parameter controlling the decay rate of the kernel. The coefficients $\alpha_{k}$ are chosen such that $\widetilde{f}(x)$ minimizes a regularized hinge loss error function on the training data set. See $[34,37]$ for a comprehensive description and an intuitive explanation.

\subsection{Logistic regression for surrogate model}

Logistic regression is a probabilistic statistical classification model that computes the probability of a point $x$ having the class label $y$. For the specific application of network connectivity, logistic regression computes the probability that a network realization is disconnected as:

$$
\widetilde{f}(x)= \begin{cases}1 & \text { if } \frac{1}{1+\exp \left(-\left(\beta_{0}+\beta_{1}^{T} x\right)\right)} \geq \xi \\ 0 & \text { otherwise }\end{cases}
$$

where $\beta_{0} \in \mathbb{R}$ is a scalar that represents an offset, and $\beta_{1} \in \mathbb{R}^{n}$ is the vector of model parameters corresponding to each element in the feature vector. Both $\beta_{0}$ and $\beta_{1}$ are learned using maximum likelihood estimation on the training data $\mathcal{D}_{\mathrm{tr}}$, and the decision threshold is set at $\xi=$ 0.5 to provide a hard classification rule that predicts the most likely class.

Intuitively, the values of $\beta_{1}$ can be thought of as relative importance factors for each element in the network. It indicates that components with larger magnitudes $\beta_{i}$ are learned to be relatively more important to network survival than others with lower magnitudes.

\section{Numerical examples}

In this section we first describe the California gas distribution network and explain how to use a fragility model to compute realistic edge failure probabilities based on an earthquake event. Next we define accuracy measures for classifying network realizations, and use these to determine the training data size needed to effectively learn a surrogate model. Finally, we demonstrate estimating network failure probability via a machine-learning-based surrogate 


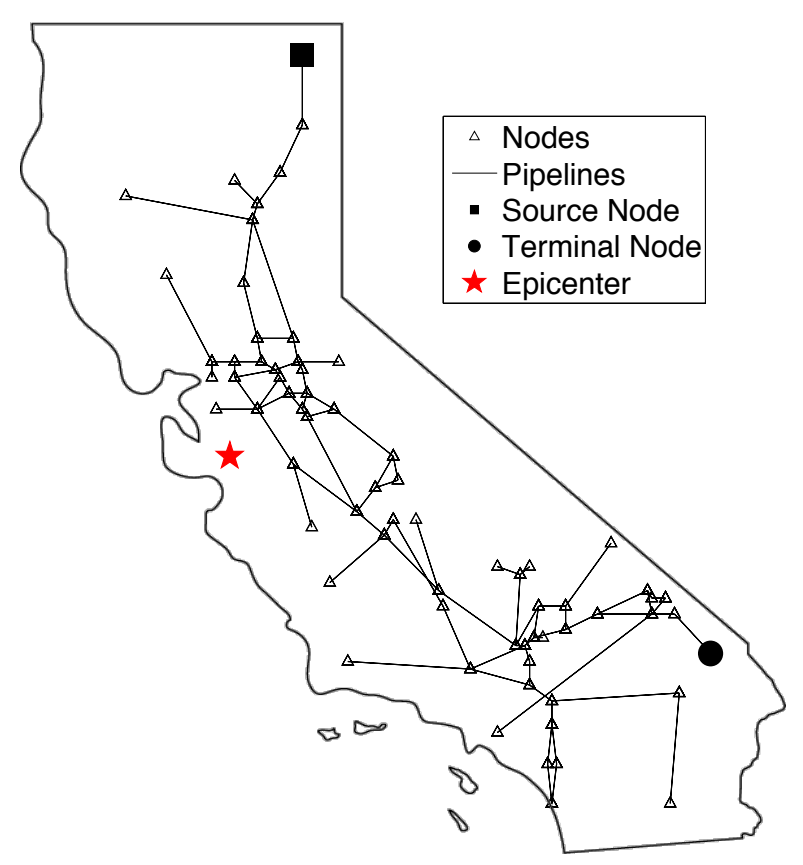

Figure 3: California gas distribution network with earthquake epicenter.

model for network connectivity on a variety of earthquake events.

\subsection{Experimental setup}

We briefly describe the benchmark infrastructure network used in this article to asses the performance of the proposed surrogate models. The California gas distribution network topology (Figure 3) is obtained from Lim et al. [38] and consists of 157 components (70 nodes and 87 bi-directional edges). In this network, each node represents a substation, and each edge represents a gas pipeline. Without loss of generality of the method, the experiments presented in this section assume that only edges are allowed to fail. A straightforward extension is to allow both edges and nodes to fail by constructing an auxiliary network with only edge failures, where each node in the original graph is replaced by two nodes connected by an edge of zero length. The source and terminal nodes used in this example are indicated in Figure 3 with a square and circle, respectively. The California gas distribution network is selected since it provides a realistic example network and is sufficiently large such that direct computation of the network failure probability via exhaustive state space computation is infeasible. Thus, approximate methods are required to estimate the probability of disconnection of the selected source and terminal nodes of the California gas distribution network.

The MATLAB source code for all methods and experiments can be accessed online [39]. All computations are conducted on a quad core 2.6 GHz MacBook Pro with 4 $\mathrm{Gb}$ of RAM. Only one core is used for computation.

\subsection{Estimating component failure with fragility models}

The robustness of each network component depends on many factors including the type of infrastructure network being considered. In this work the developed framework is applied to brittle pipeline failures under earthquake loadings. The framework can also be applied to different loading and network types by using the appropriate attenuation and fragility models.

The intensity of an earthquake dissipates as it travels through the ground from the source. The ground motion at each point in the network is determined using an attenuation law which describes how energy from an earthquake is dissipated through the ground. This can be used to calculate ground motion intensity based on the distance from the earthquake epicenter and soil parameters. The general form of an attenuation law as proposed by Goda and Hong [40] is:

$$
\ln \gamma_{j}\left(T_{n}\right)=\phi\left(M, R_{j}, \lambda_{j}, T_{n}\right)+\eta\left(T_{n}\right)+\epsilon_{i}\left(T_{n}\right) .
$$

Here $\gamma_{j}\left(T_{n}\right)$ is the ground motion intensity (e.g. PGV) at location $j$ caused by an earthquake determined for a structure with natural period, $T_{n}$. The function $\phi\left(M, R_{j}, \lambda_{j}, T_{n}\right)$ describes the attenuation of the ground motion intensity based on the earthquake magnitude $M$, the distance from the source to the $j^{\text {th }}$ location, $R_{j}$, and explanatory variables $\lambda_{j}$. The interevent residual is $\eta\left(T_{n}\right)$, and $\epsilon_{i}\left(T_{n}\right)$ is the intraevent residual. These are both assumed to be Gaussian random variables with zero mean and variance $\sigma_{\eta}^{2}$ and $\sigma_{\epsilon}^{2}$, respectively.

The attenuation law for peak ground velocity (PGV) proposed by Wang and Takada [41] that is used in this work is:

$$
\begin{aligned}
\log _{10} P G V= & 0.725 M_{j}+0.00318 H-0.519 \\
& -1.918 \log _{10}\left(D+0.334 e^{0.653 M_{j}}\right)
\end{aligned}
$$

Here $P G V$ is measured in $\mathrm{cm} / \mathrm{s}, M_{j}$ is the Japanese Meteorological Agency earthquake magnitude, $H$ is the source depth in kilometers, and $D$ is the distance between the fault plane and the site in kilometers.

Using the calculated PGV at the midpoint of the $i^{\text {th }}$ pipeline segment, it is possible to calculate probability of pipeline failure, $p_{f, i}$ based on the FEMA HAZUS-MH fragility model [42]:

$$
p_{f, i}=1-e^{-\nu_{i} l_{i}} .
$$

Here $l_{i}$ is the length of the $i^{t h}$ pipeline, and $\nu_{i}$ is the failure occurrence rate model described in the HAZUS-MH manual, and computed as

$$
\nu_{i}=\kappa\left(P G V_{i}\right)^{\tau}
$$

where $\kappa$ and $\tau$ are the scaling and exponent model parameters, respectively, and $P G V_{i}$ is the peak ground velocity at the midpoint of the $i^{t h}$ pipeline section determined by (11). 
In the event of an earthquake, edge failures are statistically dependent mainly due to spatial correlation of ground motion intensities. The correlation between the seismic intensity of pipeline $i$ and pipeline $j$ as a function of the distance in kilometers $\Delta$ obtained from Goda and Hong [40], $\rho_{\gamma_{i} \gamma_{j}}(\Delta)$ is computed as

$$
\rho_{\gamma_{i} \gamma_{j}}(\Delta)=\frac{\sigma_{\eta}^{2}}{\sigma_{\eta}^{2}+\sigma_{\epsilon}^{2}}+\rho_{\epsilon_{i} \epsilon_{j}}(\Delta) \frac{\sigma_{\epsilon}^{2}}{\sigma_{\eta}^{2}+\sigma_{\epsilon}^{2}}
$$

where

$$
\rho_{\epsilon_{i} \epsilon_{j}}(\Delta)=\exp (-0.509 \sqrt{\Delta})
$$

is the intraevent correlation, and all other terms are as defined previously. In the numerical example, PGV's are generated for each location using (11) and (15) to compute the failure probabilities in (13), which are later used to simulate component status.

\subsection{Generating data to train a machine-learning classifier}

In order to train a machine-learning-based surrogate model a representative set of examples of failed and nonfailed network realizations is required. To achieve this, first, a location of high seismic activity in the network is selected. In the case of the California gas distribution network, the epicenter of the Loma Prieta earthquake (shown in Figure 3) is selected. Next, epicenters for training earthquakes are generated by perturbing selected location with an isotropic bivariate Gaussian random distance. For this experiment, these distances were selected from a Gaussian distribution with mean 0 and variance $4 \mathrm{~km}^{2}$. Note that the greater the variance of this distribution, the less dependent the trained earthquake is on the selected area of interest.

Once the candidate training earthquake sites are selected, earthquake intensities are randomly generated at each site from a uniform distribution. For this experiment, the uniform distribution of earthquake magnitudes selected is $U(5,7)$. While earthquake magnitudes are distributed exponentially according to the Gutenberg-Richter law [43], the aim of this paper is to test the developed method under a wide verity of possible earthquake scenarios. The range of magnitudes is selected because, for this particular network, earthquakes weaker than a magnitude 5 almost never lead to network failure, while earthquakes with a magnitude greater than 7 almost always lead to network failure. Thus, earthquakes outside of this range are trivial to classify, and are not considered for training. By using this method of generating training data, the model learns the importance of individual components to the system-level survival of the network instead of the effect that a particular hazard scenario has on the survival of the network.

Training data generated from a particular earthquake scenario is split into a training set used to train a surrogate model and a hold-out test set which is used to test the predictions of the model on new data which has not previously been revealed to the classifier. The benefit of this is that the machine-learning-based surrogate model can be tested on data that is similar in structure to the data which was used for training, but was not used to construct the surrogate model. This can also be used to test for overfitting, where the classifier is trained to learn specific examples instead of learning the trends in the data. Overfitting is characterized by high accuracy on the training data which does not generalize to the hold-out test dataset. Specifically, the overlap between the training data and test data is less than $0.02 \%$, meaning that the vast majority of the data that is tested was not used to train the classifier. This overlap is a result of both training data and test data being randomly generated with replacement.

\subsection{Selecting surrogate model training data}

Machine learning classifiers are easily biased if unrepresentative training data is selected. Therefore, it is important to be able to assess how the training data influences the performance of the classifier. To measure the performance of our machine-learning-based surrogate model, three performance metrics are used. The (overall) accuracy is a weighted measure of performance of both the network failure and non-failure classes. Separately, the failure accuracy and the non-failure accuracy are performance measures that determine the accuracy of the classifier only on the network realizations that result in failed networks or non-failed networks respectively. These performance measures are calculated as:

$$
\begin{gathered}
a=\frac{T P+T N}{T P+T N+F P+F N}, \\
a_{+}=\frac{T P}{T P+F N}, \\
a_{-}=\frac{T N}{T N+F P},
\end{gathered}
$$

where $a$ is the overall accuracy, $a_{+}$is the accuracy of the network failure cases (positive class), $a_{-}$is the accuracy of the non-failure cases (negative class), $T P$ is the number of correctly predicted failure cases (true positives), $T N$ is the number of correctly predicted non-failure cases (true negatives), $F N$ is the number of falsely predicted nonfailure cases (false negatives), and $F P$ is the number of falsely predicted cases of network failure (false positives).

With the performance measures defined, it is possible to assess the size of the training dataset necessary to train an accurate model. SVM and logistic regression surrogate models are constructed using different sized training data sets ranging from 100 to 100,000 points generated according to the procedure outlined in Section 3.3. For each number of training points, ten models are learned and used for classification, and the average of the ten runs is reported. Intuitively, larger training datasets give the algorithms more examples of the relationship to be learned 
between the component states and the network connectivity status. However, it is also important to confirm that the improved accuracy is observed in the hold out test set in addition to the training data, otherwise the accuracy improvement is due to the model overfitting the training data.

We proceed by comparing the accuracy measures for each classifier on the training set and the hold-out test set of $10 \%$ the size of the training data. The resulting performance of the different models is depicted in Figures 4a, 5a, and 6a for the SVM classifiers, and 4b, 5b, and $6 \mathrm{~b}$ for the logistic regression classifiers. Negative class accuracy (cases of network survival) remains relatively high regardless of how many points are used to train the model for both the SVM and logistic regression surrogate models. However, positive class accuracy (cases of network failure) and overall accuracy increase with respect to the number of training points used for both SVM and logistic regression. This means that the more training points used to train an SVM model, the better it is able to learn a non-linear decision boundary. This is not true for logistic regression though, where additional training data does not increase the complexity of the model, but may lead to overfitting. However, for SVM, beyond 4,000 training points there is little improvement in accuracy with successive training points. For logistic regression, this plateau in positive class and overall accuracy occurs with more training data around 9,000 training points.

Since the performance on the training data is is relatively constant, and there is no amount of training data for which the classification accuracy on the test data decreases, overfitting is not occurring. Based on this information, we select an SVM surrogate model constructed with 4,000 training points and a logistic regression surrogate model trained with 9,000 training points for this specific network since this is sufficiently complex to capture the network structure while not being too complex to negate the gain in computational time due to an excessive training data. The total time required to train the logistic regression-based surrogate model is 42.29 seconds, while the total time required to train the SVM-based surrogate model is 4.38 seconds. This includes the time to generate enough training data to construct each model. For larger or more complex networks, training a surrogate model may require more training data, while for smaller or simpler networks it may be possible to use fewer training data.

\subsection{Estimating network failure probability}

To simulate a realistic earthquake scenario, the 1989 Loma Prieta earthquake in Santa Cruz, CA is used for this numerical example. The earthquake was measured to have magnitude $M=6.9$ with an epicenter $19 \mathrm{~km}$ below the surface located at the point indicated with a star in Figure 3. Additionally, six other earthquake scenarios ranging in intensity from $M=5.0$ to $M=7.0$ given in 1 with the

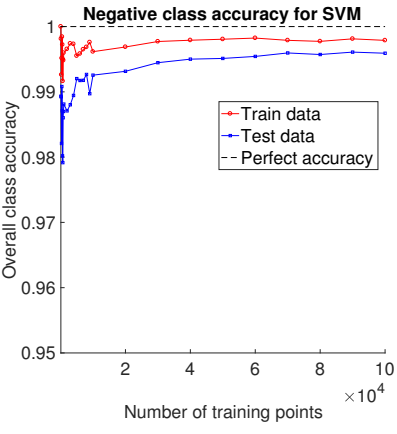

(a)

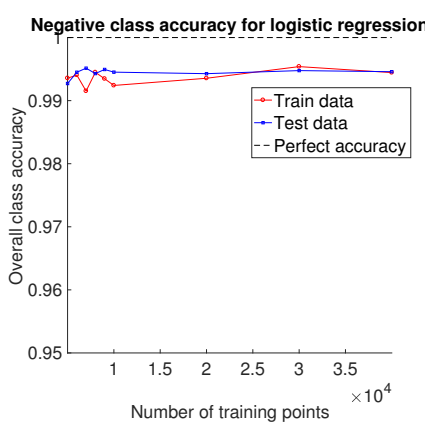

(b)
Figure 4: Testing and training accuracy for cases of non-failure - (a) SVM and (b) logistic regression.

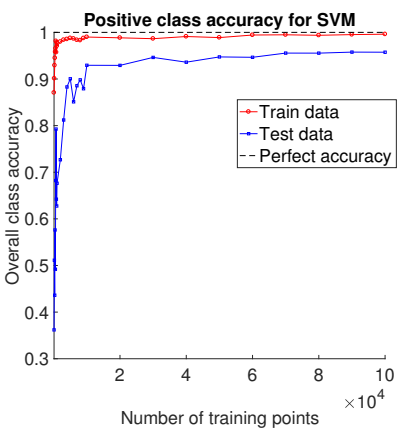

(a)

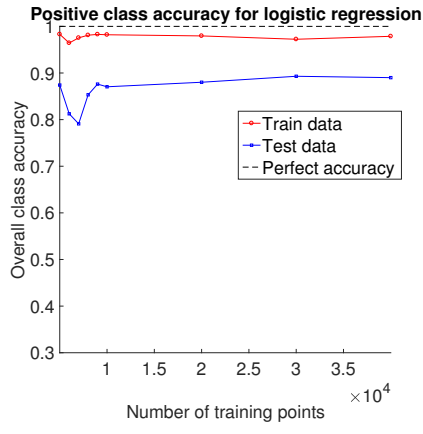

(b)
Figure 5: Testing and training accuracy for cases of network failure - (a) SVM and (b) logistic regression.

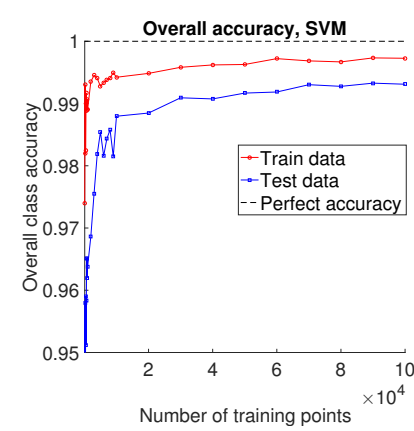

(a)

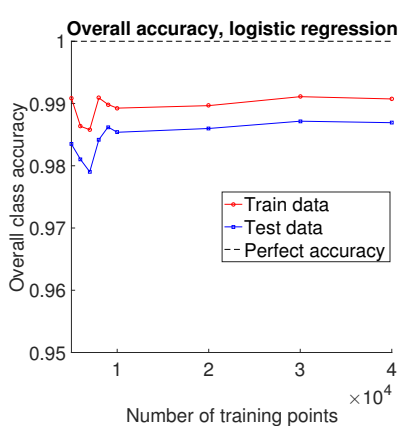

(b)
Figure 6: Overall testing and training accuracy - (a) SVM and (b) logistic regression. 


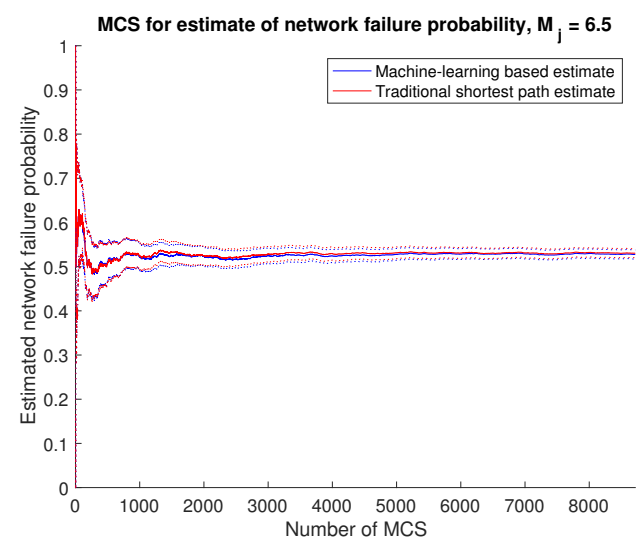

Figure 7: Convergence of MCS estimate using both traditional DFS connectivity check and SVM-based surrogate model. Network failure estimate as well as COV is shown at each step in the simulation.

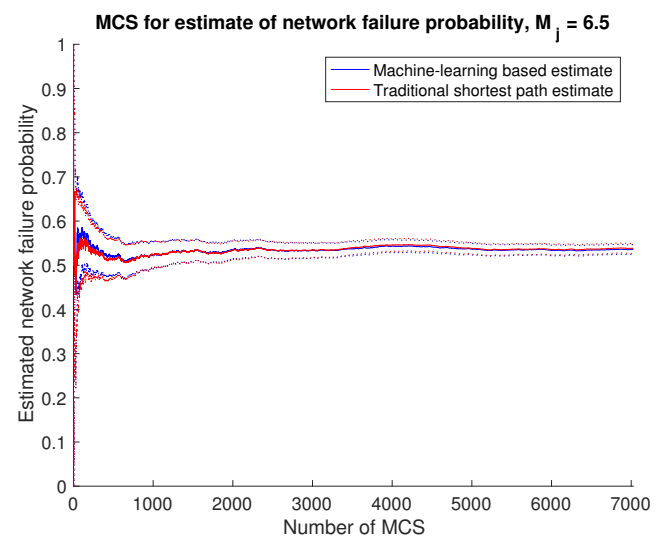

Figure 8: Convergence of MCS estimate using both traditional DFS connectivity check and the logistic-regression-based surrogate model. Network failure estimate as well as COV is shown at each step in the simulation.

same epicenter and depth as the Loma Prieta earthquake are used to assess the algorithm.

The PGV and spatial correlation are computed at the midpoint of each edge. Using the attenuation law and correlation described in Section 3.2, as well as the segment length and PGV, the edge failure probability is computed using (13) and (14), with $\kappa=0.0001$ and $\tau=2.5$. The result of the Loma Prieta earthquake scenario is that the majority of edges have a low failure probability while edges in close proximity to the earthquake epicenter have a high failure probability.

The network failure probability is estimated using both the SVM and logistic regression surrogate models for the Loma Prieta earthquake scenario, as well as others using the same epicenter and spatial correlation as the Loma Prieta earthquake, but with different intensity. The resulting estimates of network failure probability and convergence time are summarized in Table 1 . The accuracies of SVM and logistic regression are compared in Table 2 . In most cases both the positive and negative class accuracy, as well as the overall accuracy are very high for both methods.
This indicates good performance of the surrogate models when predicting network failure. The exception is that for very weak earthquakes, some network realizations corresponding to network failure are not identified as such by the SVM surrogate model, while the logistic regression surrogate model is able to detect these realizations as network failures and estimate the probability of network failure even when it is very low. This is left as an observation since there is no clear reason that SVM should preform worse than logistic regression in this case. Further research is required to identify if with different training data, a kernel SVM surrogate model would achieve higher accuracy than the logistic regression surrogate model.

The gains in computation time are significant as well. The logistic regression surrogate model achieves a two order of magnitude reduction in computation time, while the SVM classifier only achieves a 4 -fold reduction in computation time.

The convergence plot of the SVM surrogate model estimating the network failure probability of a magnitude 6.5 earthquake with the same epicenter as the Loma Prieta earthquake is shown in Figure 7. The convergence of the logistic regression surrogate model on the same earthquake is shown in Figure 8. This shows the quick convergence of both the SVM and logistic regression surrogate modelbased estimates of network failure probability to the true network failure probability.

\subsection{Robustness to earthquake locations}

The SVM model is learned using training data from earthquakes close to the epicenter of the Loma Prieta earthquake. This is because the location of the fault line is known in advance, and thus the hazard risk is known in advance. This means that earthquakes with epicenters far from this location may not be considered when constructing the surrogate model. However, it is important that this method is also applicable in cases when the earthquake occurs far away from the earthquake epicenters used to generate the training data.

To investigate the ability of the SVM surrogate model to generalize to earthquakes at different locations, we test the SVM and logistic regression surrogate models for earthquakes with epicenters of varying distances from the epicenters of the earthquakes used to generate the training data. The locations tested, as well as the training earthquakes are shown in Figure 9. For simplicity, all earthquakes have a magnitude of 6.5. The resulting network failure probability estimates and corresponding accuracies are presented in Table 3 .

The results in Table 3 show that for most cases the SVM surrogate model generalizes to earthquakes that are not in the proximity of the earthquakes used to generate training data. While some degradation in failure estimation quality is observed, the network failure probability estimates are accurate to $84 \%$ or higher. However, this is not the case for the logistic regression surrogate model. The performance of the logistic regression surrogate model 


\begin{tabular}{c|cccccc}
\hline & \multicolumn{2}{|c}{ Depth First Search } & \multicolumn{2}{c}{ Support Vector Machine } & \multicolumn{2}{c}{ Logistic Regression } \\
$M_{j}$ & $\hat{P}\left(Y_{s, t}=1\right)$ & $T_{D F S}(\mathrm{~s})$ & $\hat{P}\left(Y_{s, t}=1\right)$ & $T_{S V M}(\mathrm{~s})$ & $\hat{P}\left(Y_{s, t}=1\right)$ & $T_{L R}(\mathrm{~s})$ \\
\hline 5.0 & 0.0004 & 19.19 & 0.0004 & 4.01 & 0.0006 & 0.1528 \\
5.5 & 0.0056 & 4.69 & 0.0055 & 1.03 & 0.0060 & 0.0343 \\
6.0 & 0.0714 & 0.36 & 0.0712 & 0.08 & 0.0702 & 0.0049 \\
6.5 & 0.5263 & 0.53 & 0.5292 & 0.13 & 0.5253 & 0.0054 \\
6.9 & 0.9473 & 0.03 & 0.9727 & 0.01 & 0.9590 & 0.0006 \\
7.0 & 0.9900 & 0.39 & 0.9907 & 0.11 & 0.9829 & 0.0032 \\
\hline
\end{tabular}

Table 1: Comparison of estimated network failure probability and computation time $T_{D F S}, T_{S V M}$, and $T_{L R}$ using DFS, SVM, and logistic regression, respectively.

\begin{tabular}{c|cccccc}
\hline & \multicolumn{3}{|c}{ Support Vector Machine } & \multicolumn{3}{c}{ Logistic Regression } \\
$M_{j}$ & $a$ & $a_{+}$ & $a_{-}$ & $a$ & $a_{+}$ & $a_{-}$ \\
\hline 5.0 & 0.999 & 0.511 & 0.999 & 0.999 & 0.860 & 0.999 \\
5.5 & 0.997 & 0.718 & 0.998 & 0.998 & 0.883 & 0.999 \\
6.0 & 0.981 & 0.885 & 0.988 & 0.986 & 0.916 & 0.992 \\
6.5 & 0.966 & 0.968 & 0.965 & 0.962 & 0.963 & 0.961 \\
6.9 & 0.988 & 0.998 & 0.815 & 0.982 & 0.988 & 0.833 \\
7.0 & 0.999 & 0.999 & 0.929 & 0.989 & 0.990 & 0.897 \\
\hline
\end{tabular}

Table 2: Comparison of logistic-regression-based and SVM-based network failure probability estimate for the Loma Prieta earthquake.

\begin{tabular}{ccccccc}
\hline & & Depth First Search & \multicolumn{2}{c}{ Support Vector Machine } & \multicolumn{3}{c}{ Logistic Regression } \\
Epicenter & Distance $(k m)$ & $\hat{P}\left(Y_{s, t}=1\right)$ & $\hat{P}\left(Y_{s, t}=1\right)$ & $a$ & $\hat{P}\left(Y_{s, t}=1\right)$ & $a$ \\
\hline 1 & 0.0 & 0.5263 & 0.5292 & 0.996 & 0.5253 & 0.962 \\
2 & 141 & 0.9533 & 0.9519 & 0.997 & 0.9380 & 0.990 \\
3 & 375 & 0.3622 & 0.3166 & 0.837 & 0.010 & 0.642 \\
4 & 266 & 1.000 & 0.8394 & 0.839 & 0.175 & 0.175 \\
5 & 500 & 0.999 & 1.000 & 0.999 & 0.201 & 0.210 \\
\hline
\end{tabular}

Table 3: Network failure probability estimate and corresponding accuracy of magnitude 6.5 earthquakes with differing epicenters using both SVM and logistic regression surrogate models. Epicenter locations shown in Figure 9. The distance in kilometers to the epicenter of the Loma Prieta earthquake (epicenter 1) is given. 


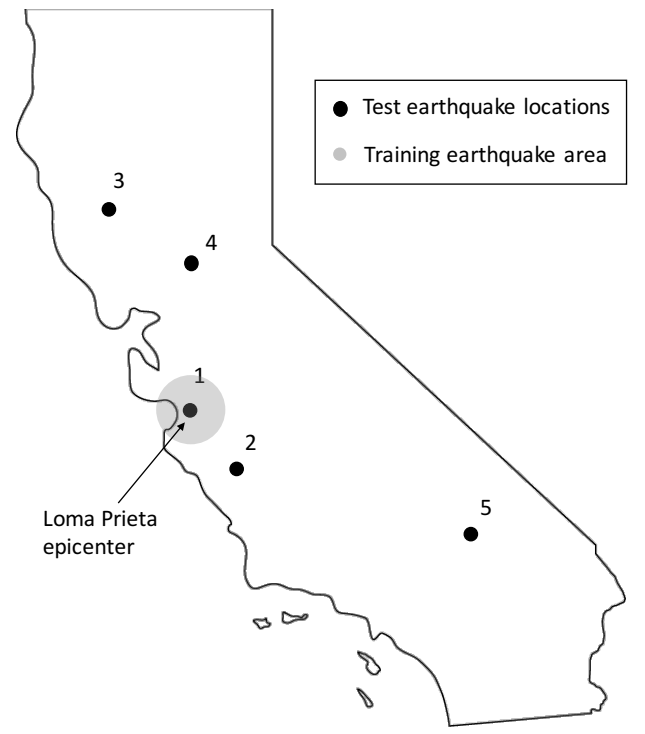

Figure 9: Locations of epicenters of earthquake scenarios considered. Scenario numbers correspond earthquakes considered in Table 3. Earthquake 1 is coincident with the Loma Prieta earthquake epicenter. Grey circle indicates the area where training data was sampled from to construct the surrogate models.

deteriorates significantly as the data is selected from further from the earthquakes used to generate training data. This indicates that the logistic regression surrogate model is not as generalizable as the SVM surrogate model, which is more robust to different earthquake epicenters. The robustness of the kernel SVM surrogate model is a result of the non-linearity introduced by the kernel function. This allows for the kernel SVM-based surrogate model to learn a non-linear decision boundary, which results in a more generalizable surrogate model than that learned by the linear logistic regression surrogate model.

\section{Conclusion}

The methods and corresponding numerical simulations presented in this paper indicate that it is possible to use machine-learning-based surrogate models in place of exact connectivity checks when conducting MCS to estimate the probability of network failure following a disaster event such as an earthquake. The methods are able to produce estimates of the probability of network failure that are very similar to traditional MCS methods but are up to two orders of magnitude faster. These results demonstrate that machine learning methods may be used to construct surrogate models for network failure, which may eventually be used in place of exact connectivity checks to greatly improve the speed of convergence of MCS estimates on network failure probability for stochastic networks.

One limitation of the presented method is that, while efforts were made to make the method generalizable, it is not equally accurate at predicting network disconnections for all hazards. Further research will focus on collecting more representative training data. This may be done using importance sampling $[44,45]$ or other sampling techniques that collect training data from a broader range of potential hazards.

One extension to this specific work is the development of surrogate models that can be used in conjunction with MCS to estimate the probability of network disconnections for the $k$-terminal reliability, or even the all-terminal reliability problem, which are more complex problems than the one addressed in this paper [46, 47]. Qualitatively, to achieve this, the class label would change from connected and disconnected to $k$-nodes connected and at least one disconnection. In previous work, the authors considered this problem and used spectral graph clustering to identify densely connected sets of nodes, and trained a machine learning based surrogate model to predict network disconnections between these clusters of nodes instead of a source and terminal node [48]. However, this requires specific sets of nodes to be selected via clustering algorithms. Learning a surrogate model for the more general $k$-terminal connectivity problem may prove to be challenging since the network structure cannot be exploited as was the case in [48]. This, in addition to work to focus on gaining a deeper understanding of what effect different training data earthquake epicenters have on the quality of prediction, would serve to further the methods developed in this paper.

Finally, the machine-learning-based surrogate model presented in this article may be used to develop more efficient sampling methods. For example, the surrogate model of network connectivity could be combined with methods such as importance sampling, subset simulations, and Monte Carlo Markov Chain (MCMC) sampling, among others, to improve their computation time $[49,50,51]$.

\section{Acknowledgements}

This material is based upon work supported by the National Science Foundation under grant number CMMI 1031318. The second author would like to acknowledge support by the National Research Foundation of Korea (NRF) Grant (No. 2015R1A5A7037372) funded by the Korean Government (MSIP) and the Integrated Research Institute of Construction and Environmental Engineering at Seoul National University.

\section{References}

[1] Michael Bruneau, Stephanie E. Chang, Ronald T. Eguchi, George C. Lee, Tom D. O'Rourke, Andrei Reinhorn, M. Shinozuka, Kathleen Tierney, William A. Wallace, and Detlof von Winterfeldt. A framework to quantitatively assess and enhance the seismic resilience of communities. Earthquake Spectra, 19(4):733-752, 2003.

[2] Arjen Boin and Allan McConnell. Preparing for critical infrastructure breakdowns: The limits of crisis management and the need for resilience. Journal of Contingencies and Crisis Management, 15(1):50-59, 2007.

[3] Junho Song and Seung-Yong Ok. Multi-scale system reliability analysis of lifeline networks under earthquake hazards. Earthquake Engineering and Structural Dynamics, 39:259-279, 2009. 
[4] Eric D. Vugrin, Drake E. Warren, Mark A. Ehlen, and R. Chris Camphouse. Sustainable and Resilient Critical Infrastructure Systems: Simulation, Modeling, and Intelligent Engineering, chapter A Framework for Assessing the Resilience of Infrastructure and Economic Systems, pages 77-116. Springer Heidelberg, Heidelberg, 2010.

[5] Dorothy A. Reed, Kailash C. Kapur, and Richard D. Christie. Methodology for assessing the resilience of networked infrastructure. IEEE Systems Journal, 3(2):174-180, June 2009.

[6] Armen Der Kiureghian and Junho Song. Multi-scale reliability analysis and updating of complex systems by use of linear programming. Reliability Engineering ES System Safety, 93:288297,2008

[7] Joung-Joo Lee, Junho Song, Paolo Gardoni, and Huy-Woo Lim. Post-hazard flow capacity of bridge transportation network considering structural deterioration of bridges. Structure and Infrastructure Engineering, 7(7-8):509-521, 2011.

[8] Michael O. Ball. Computational complexity of network reliability analysis: An overview. IEEE Transactions on Reliability, 35(3):230-239, 1986.

[9] Sieteng Soh and Suresh Rai. An efficient cutset approach for evaluating communication-network reliability with heterogeneous link-capacities. IEEE Transactions on Reliability, 54(1):133-144, 2005.

[10] Jason L. Cook and Jose E. Ramirez-Marquez. Two-terminal reliability analyses for a mobile ad hoc wireless network. Reliability Engineering \& System Safety, 92(6):821 - 829, 2007.

[11] Sarintip Satitsatian and Kailash C. Kapur. An algorithm for lower reliability bounds of multistate two-terminal networks. IEEE Transactions on Reliability, 55(2):199-206, 2006.

[12] Timothy B. Brecht and Charles J. Colbourn. Lower bounds on two-terminal network reliability. Discrete Applied Mathematics, 21(3):185 - 198, 1988.

[13] Chin-Chia Jane and Yih-Wenn Laih. A practical algorithm for computing multi-state two-terminal reliability. IEEE Transactions on Reliability, 57(2):295-302, 2008.

[14] Luigi Fratta and Ugo G. Montanari. A boolean algebra method for computing the terminal reliability in a communication network. IEEE Transactions on Circuit Theory, 20(3):203-211, 1973.

[15] Shawqi Kharbash and Wenye Wang. Computing two-terminal reliability in mobile ad hoc networks. In Wireless Communications and Networking Conference, pages 2831-2836. IEEE, 2007.

[16] Corinne Lucet and Jean-François Manouvrier. Exact methods to compute network reliability. In Statistical and Probabilistic Models in Reliability, pages 279-294. Springer, 1999.

[17] Fred S. Roberts, Frank Hwang, and Clyde L. Monma. Reliability of computer and communication networks, volume 5. American Mathematical Society, 1991.

[18] J. Scott Provan and Michael O. Ball. The complexity of counting cuts and of computing the probability that a graph is connected. SIAM Journal on Computation, 12(4):777-788, 1983.

[19] Arnie Rosenthal. Computing the reliability of complex networks. SIAM Journal on Applied Mathematics, 32(2):384-393, 1977.

[20] David R. Karger. A randomized fully polynomial time approximation scheme for the all-terminal network reliability problem. SIAM Review, 43(3):499-522, 2001.

[21] Chin Yang Lee. An algorithm for path connections and its applications. IRE Transactions on Electronic Computers, (3):346365, 1961 .

[22] Robert E. Tarjan. Depth-first search and linear graph algorithms. SIAM Journal on Computing, 1(2):146-160, 1972.

[23] Edsger Dijkstra. A note on two problems in connexion with graphs. Numerische Mathematik, 1(1):269-271, 1956.

[24] Stuart E. Dreyfus. An appraisal of some shortest-path algorithms. Operations research, 17(3):395-412, 1969.

[25] Shimon Even and Robert E. Tarjan. Network flow and testing graph connectivity. SIAM Journal on Computing, 4(4):507-518, 1975 .
26] Hong-shuang Li, Zhen-zhou Lü, and Zhu-feng Yue. Support vector machine for structural reliability analysis. Applied Mathematics and Mechanics, 27:1295-1303, 2006.

[27] J-M Bourinet, François Deheeger, and Maurice Lemaire. Assessing small failure probabilities by combined subset simulation and support vector machines. Structural Safety, 33(6):343-353, 2011.

28] Raphael E. Stern, Junho Song, and Daniel B. Work. Machine learning-based surrogate models for network reliability analysis. $17^{\text {th }}$ IFIP WG 7.5 July 3-7, 2014.

[29] Yoav Freund and Robert E. Schapire. A short introduction to boosting. Journal of Japanese Society for Artificial Intelligence, 14(5):771-780, 1999.

[30] Hyun-Woo Lim and Junho Song. Efficient risk assessment of lifeline networks under spatially correlated ground motions using selective recursive decomposition algorithm. Earthquake Engineering \&f Structural Dynamics, 41(13):1861-1882, 2012.

[31] Richard M. Karp, Michael Luby, and Neal Madras. Monte-carlo approximation algorithms for enumeration problems. Journal of Algorithms, 10(3):429-448, 1989.

[32] Norman P. Hummon and Patrick Doreian. Computational methods for social network analysis. Social Networks, 12(4):273 $-288,1990$.

[33] Gerardo Rubino and Bruno Tuffin. Rare Event Simulation using Monte Carlo Methods. John Wiley \& Sons, Chichester, UK, 2009.

[34] Bernhard Scholkopf and Alexander J. Smola. Learning with Kernels: Support Vector Machines, Regularization, Optimization, and Beyond. MIT Press, Cambridge, MA, USA, 2001.

[35] Chih-Wei Hsu, Chih-Chung Chang, Chih-Jen Lin, et al. A practical guide to support vector classification. Technical report, National Taiwan University, Department of Computer Science, 2003.

[36] Christopher Burges. A tutorial on support vector machines for pattern recognition. Data Mining and Knowledge Discovery, 2(2):121-167, 1998.

[37] Nello Cristianini and John Shawe-Taylor. An introduction to support vector machines and other kernel-based learning methods. Cambridge university press, 2000.

[38] Huy-Woo Lim, Junho Song, and Kurtz Nolan. Seismic reliability assessment of lifeline networks using cluster-based multiscale approach. Earthquake Engineering \& Structural Dynam$i c s$, in print. doi:10.1002/eqe.2472.

[39] Raphael Stern. California gas distribution network source code, 2015.

[40] Keisuke Goda and Han-Ping Hong. Spatial correlation of peak ground motions and response spectra. Bulletin of the Seismological Society of America, 98(1):354-365, 2008.

[41] Min Wang and Tsuyoshi Takada. Macrospatial correlation model of seismic ground motions. Earthquake Spectra, 21(4):1137-1156, 2005.

[42] FEMA. HAZUS MH-MR3 Technical Manual. Federal Emergency Management Agency, Washington, DC, 2008.

[43] Beno Gutenberg. Seismicity of the earth and associated phenomena. Read Books Ltd, 2013.

[44] Radford M Neal. Annealed importance sampling. Statistics and Computing, 11(2):125-139, 2001.

[45] Peter W Glynn and Donald L Iglehart. Importance sampling for stochastic simulations. Management Science, 35(11):13671392, 1989.

[46] Charles J Colbourn and CJ Colbourn. The combinatorics of network reliability, volume 200. Oxford University Press New York, 1987.

[47] R Kevin Wood. Factoring algorithms for computing kterminal network reliability. IEEE Transactions on Reliability, 35(3):269-278, 1986.

[48] Raphael E Stern, Junho Song, and Daniel B Work. Network reliability analysis for cluster connectivity using adaboost. In Proceedings of the 12th International Conference on the Applications of Statistics and Probability in Civil Engineering. Vancouver, Canada, July 12-15, 2015. 
[49] Nolan Kurtz and Junho Song. Cross-entropy-based adaptive importance sampling using gaussian mixture. Structural Safety, 42:35-44, 2013.

[50] Ziqi Wang and Junho Song. Cross-entropy-based adaptive importance sampling using von mises-fisher mixture for high dimensional reliability analysis. Structural Safety, 59:42-52, 2016.

[51] Siu-Kui Au and James L Beck. Estimation of small failure probabilities in high dimensions by subset simulation. Probabilistic Engineering Mechanics, 16(4):263-277, 2001. 